\title{
A randomized intervention trial to reduce mechanical exposures in the Colombian flower industry
}

\author{
Barrero LH ${ }^{\mathrm{a},{ }^{*}}$, Ceballos $\mathrm{C}^{\mathrm{b}}$, Ellegast $\mathrm{R}^{\mathrm{c}}$, Pulido JA ${ }^{\mathrm{a}}$, Monroy $\mathrm{M}^{\mathrm{a}}$, Berrio $\mathrm{S}^{\mathrm{a}}$ and Quintana $\mathrm{LA}^{\mathrm{a}}$ \\ ${ }^{\mathrm{a}}$ Centro de Estudios de Ergonomía, Department of Industrial Engineering, School of Engineering, Pontificia \\ Universidad Javeriana, Carrera 7 \# 40-62, Ingenería Bogotá, Colombia \\ ${ }^{\mathrm{b}}$ A.R.P. SURA, Avenida El Dorado \# 68B-85 piso 9 Bogotá, Colombia \\ ${ }^{c}$ IFA - Institute for Occupational Safety and Health of the German Social Accident Insurance, Alte Heerstr. 111 \\ 53757 Sankt Augustin, Germany
}

\begin{abstract}
Evidence on the effectiveness of ergonomic interventions to reduce mechanical demands and upper-extremity MSDs is scarce in agriculture. We conducted an intervention to reduce mechanical exposures during manual flower cutting through job rotation, education and reduction of force requirements. One-hundred and twenty workers (20 to 60 years old; $89 \%$ women) from six companies that cultivate roses participated in this study. Three companies were randomly assigned to control and intervention groups. We studied changes between baseline and follow-up in self-reported effort and upper-extremity postures, kinematics and muscular activity. Most of the observed changes were moderate for both groups. The intervention group showed differential improvements compared to the control group for the maximum wrist radial deviation and forearm pronation, and acceleration of the forearm supination-pronation and elbow flexion-extension; and the muscular activity of the flexor and extensor carpi radialis and the flexor carpi ulnaris. However, we also observed that the maximum ulnar deviation, velocity of the wrist flexion-extension and muscular activity of the extensor carpi ulnaris improved more in the control group. These mixed results may be related to limited time for intervention adjustment, and uncontrolled task changes in the control group. Future research should address these issues and test other solutions.
\end{abstract}

Keywords: Randomized trial, intervention, mechanical exposures, agriculture

\section{Introduction}

There is only moderate evidence regarding the effectiveness of ergonomic interventions in preventing high mechanical demands and upper-extremity MSDs [1-3]. The evidence is particularly scarce in agriculture, in spite of the millions of people dedicated to this demanding work [4]. In Colombia, the flower industry, which employs over 90000 workers in production areas, accounts for most recorded cases of musculoskeletal disease [5]. In this industry, the flower cutting task would be among the most demanding [6]. We report preliminary results of an intervention aiming to reduce mechanical exposures among manual flower cutting workers of the Colombian flower industry.

\section{Methods}

\subsection{Population}

One-hundred and twenty workers (age ranging from 20 to $60 ; 89 \%$ women) from six companies that cultivate roses in the Bogota plateau participated in this study. Workers were required to have at least one year of experience in the job and dedicate at least $10 \%$ of the time to the flower cutting task. In the Co-

\footnotetext{
${ }^{*}$ Corresponding author. E-mail: lopehugo@javeriana.edu.co
} 
lombian flower industry, flower cutting is typically executed manually using a pruner. The task is completed during the first 3 to 4 hours of the work shift in greenhouses at frequencies near 4 to 8 cuts per minute during low demand season [3] (Figure 1).

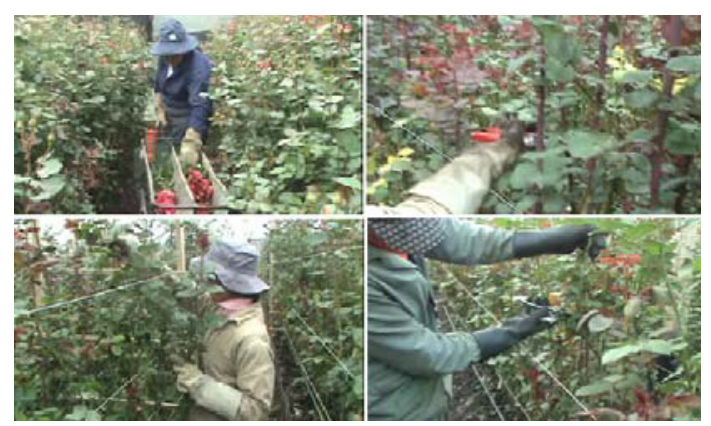

Fig 1. Manual flower cutting using a pruner

Fifty-three persons were lost to follow-up and had to be replaced. Losses were mostly due to job change; and were younger, had less seniority in the job, were more frequently men and were healthier in comparison to persons staying in the study. Procedures were approved by the ethical committee of the School of Medicine of Pontificia Universidad Javeriana, Bogota, Colombia.

\subsection{Study design}

Three companies were randomly assigned to be part of the control and intervention groups. There were no significant differences between persons in the control and intervention groups at baseline, except for the height of the workers (Table 1). Baseline and follow-up measurements were made in years 2009 and 2010, respectively. The intervention took place between December 2009 and March 2010. Changes in the exposure variables between baseline and follow-up during flower cutting were compared for the intervention and control groups while adjusting for company clusters. We measured potential effects on self-reported effort (Borg Scale); postures, velocities and accelerations in the flexion-extension (F-E) and ulnar-radial (U-R) directions of the wrist, supination-pronation (S-P) direction of the forearm, and flexion-extension of the elbow (Cuela System, Germany); and muscle activity of the forearm muscles and biceps as measured by the normalized RMS signal (Biometrics, USA).

\subsection{Intervention}

The intervention addressed force requirements of the task through the implementation of a tool main-

Table 1

Baseline differences between control and intervention groups

\begin{tabular}{|c|c|c|c|}
\hline & Control $(n=60)$ & Intervention $(\mathrm{n}=60)$ & $\mathbf{p}$ \\
\hline Age (years) & $36(8.6)$ & $37.9(9.3)$ & 0.4107 \\
\hline Job seniority (years) & $5.8(5.6)$ & $8.4(4.8)$ & 0.2456 \\
\hline Height (m) & $1.6(0.1)$ & $1.5(0.1)$ & 0.0053 \\
\hline Mass (Kg) & $59.2(8.8)$ & $58.6(8.3)$ & 0.5449 \\
\hline $\operatorname{BMI}\left(\mathrm{Kg} / \mathrm{m}^{2}\right)$ & $24.1(3.7)$ & $24.7(3.3)$ & 0.1076 \\
\hline Women $(\%)$ & 83.3 & 85 & 0.7880 \\
\hline \multicolumn{4}{|l|}{ Symptoms } \\
\hline Carpal tunnel syndrome (\%) & 28.3 & 43.3 & 0.0616 \\
\hline Epicondylitis (\%) & 11.7 & 28.3 & 0.2394 \\
\hline De Quervain's disease (\%) & 23.3 & 16.7 & 0.3805 \\
\hline Trigger finger $(\%)$ & 3.3 & 0.0 & - \\
\hline
\end{tabular}

tenance program; tasks' durations through a rotation program; and workstyle through an education program to promote less pronation and supination during flower cutting. Although the control group did not receive any intervention from our group, we could not prevent companies from executing prevention actions. The intervention approach was participative and defined with companies' representatives and workers; and considered companies' investment possibilities and productivity. Compliance was evaluated 
for the work style through one or more visits to the workplace.

\section{Results}

Most of the observed exposures had only moderate changes for both groups. We observed improvements in the intervened workers while the control group remained mostly unchanged or became more exposed for the maximum radial deviation, maximum pronation, maximum acceleration in the S-P direction and mean acceleration of the elbow F-E. However, the opposite was true for the maximum ulnar deviation and velocity of the wrist F-E. Other variables showed improvements in both groups including velocity and acceleration of the U-R deviation. Also, we observed more elbow flexion in the intervention group, which has been related to reduced risk of musculoskeletal upper-extremity symptoms in this population [7] (Figure 2). Lastly, muscle activities were only slightly lower in the intervention group for the flexor and extensor carpi radialis and the flexor carpi ulnaris, but not for the extensor carpi ulnaris which showed elevated muscle activities in the intervened workers.

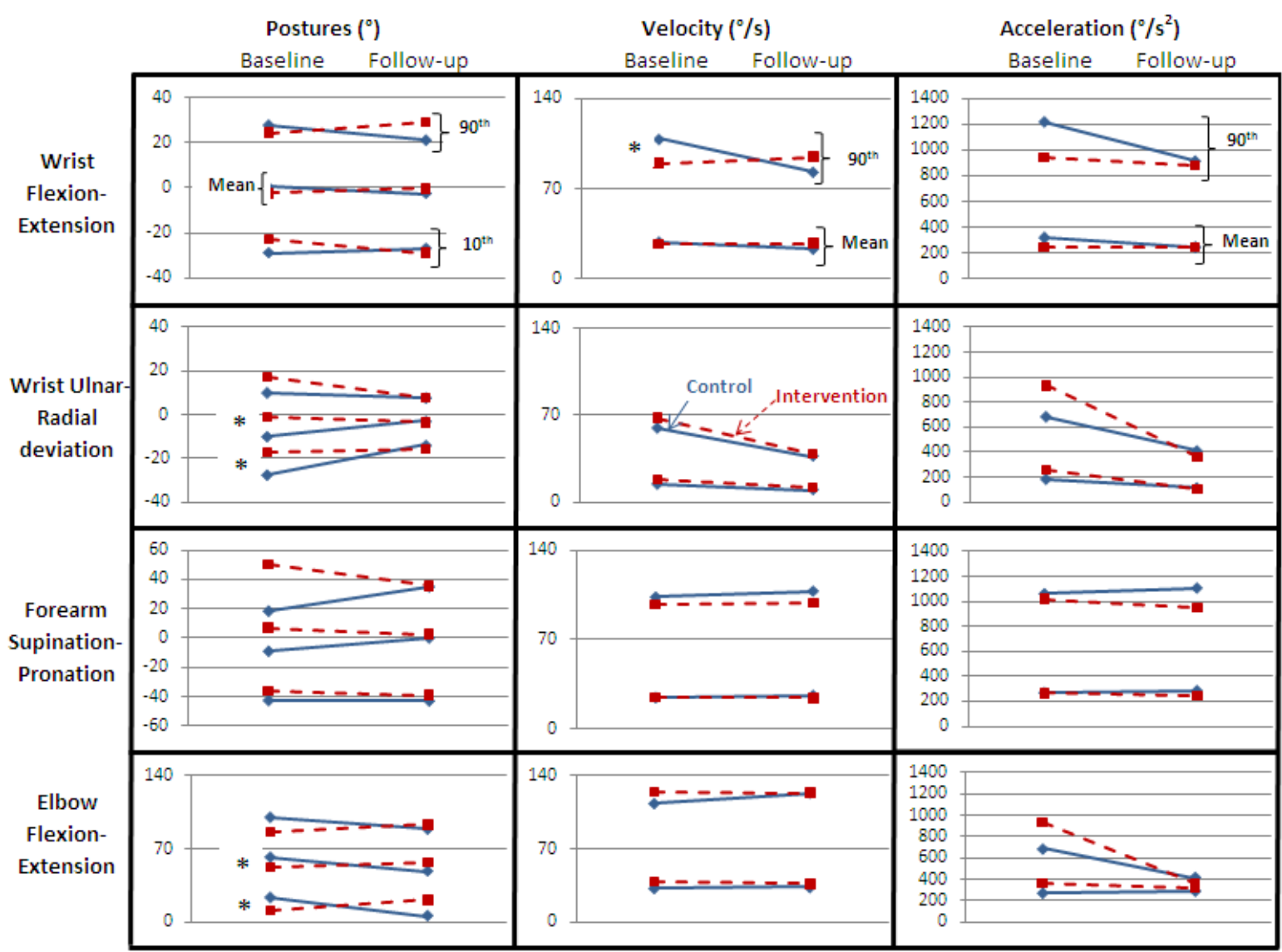

Fig 2. Postures, velocities and accelerations of wrist, forearm and elbow, with mean $90^{\text {th }}$ and $10^{\text {th }}$ percentile

*Significant tests $(\mathrm{p}<0.05)$ for the significance of the interaction between group and time are presented. Posture negative values correspond to wrist extension, ulnar deviation and forearm supination.

\section{Conclusions}

This study is the first attempt in the Colombian flower industry to document changes resulting from a randomized field intervention. Although these are preliminary results, it appears that the intervention resulted in only moderate and mixed exposure changes. These results may be related to insufficient 
compliance, limited time for intervention adjustment; and still uninvestigated potential differences between groups including discrepancies in production rates and the possibility of potential changes to the tasks in the control group, factors that are difficult to keep under control in a field trial. Future research should address these issues and test other potential solutions.

\section{Acknowledgements}

The authors thank A.R.P. SURA for funding the study. We are also grateful to the companies and workers who enthusiastically participated in this study.

\section{References}

[1] Brewer, S., et al., Workplace interventions to prevent musculoskeletal and visual symptoms and disorders among computer users: A systematic review. Journal of Occupational Rehabilitation, 2006. 16(3): p. 325-58

[2] Verhagen, A.P., et al., Exercise proves effective in a systematic review of work-related complaints of the arm, neck, or shoulder. Journal of Clinical Epidemiology, 2007. 60(2): p. 110-7.

[3] Barrero, et al., Physical workloads of the upper-extremity among workers of the Colombian flower industry. Submitted to Applied Ergonomics, 2011.

[4] Fathallah, F.A., Musculoskeletal disorders in labor-intensive agriculture. Appl Ergon, 2010. 41: p. 738-43.

[5] Ministerio de la Protección Social, Enfermedad Profesional en Colombia 2003-2005. 2007, Fondo Nacional de Riesgos Profesionales: Bogotá.

[6] Barrero, L.H., et al. Upper-extremity muscle activity during flowers cutting and packing in a Colombian working population. in PREMUS. 2010. Angers, France.

[7] Barrero, L.H., et al. Mechanical determinants of carpal tunnel syndrome in a population of the Colombian flower industry. in PREMUS. 2010. Angers, France. 